\title{
The Science of Phylogenetic Systematics: Explanation, Prediction, and Test
}

\author{
Arnold G. Kluge ${ }^{1}$ \\ Museum of Zoology, University of Michigan, Ann Arbor, Michigan 48109
}

Accepted October 25, 1999

When the concept of homology is operationalized with synapomorphy and tested with character congruence, homology and homoplasy are treated as a complement relation, $a$ and not- $a$, respectively. This leaves homoplasy to be defined nominally, something like operational "error" in the inference of homology. In choosing the most severely tested and least disconfirmed cladogram, those errors are minimized, and the power of that cladogram to explain synapomorphies, as inherited from the same common ancestral condition, is correspondingly maximized. Tests of predictions of homoplasy can lead to the elimination of those kinds of error. The complementary relationship between homology and homoplasy is considered one of reciprocal clarification, not epistemological dependence. $\odot 1999$ The Willi Hennig Society

\section{INTRODUCTION}

The question prompting this discussion of homology and homoplasy is whether synapomorphy can be explained deductively and be a test of sister-group relationships. ${ }^{2}$ At recent Willi Hennig Society meetings,

${ }^{1}$ E-mail: akluge@umich.edu.

${ }^{2} \mathrm{My}$ use of the term synapomorphy is a literal one, "a sharedderived character state," and such a definition continues to allow for characters later judged to be homoplasious (wing, as in bats and in birds). Also, strictly speaking, my use of the term homology does
Kirk Fitzhugh (e.g., Fitzhugh, 1998) argued that synapomorphy cannot constitute disconfirming evidence of competing cladograms, because synapomorphy is the effect a cladogram is intended to explain. In other words, synapomorphy as evidence does not meet the scientific standard of independence, and therein, according to Fitzhugh, lies a fallacy. Fitzhugh's conjecture of non-independence may be considered a particularly serious challenge to phylogenetic systematics, because it denies that the most severely tested and least disconfirmed cladogram can also maximize explanatory power (sensu Farris, 1983). According to Fitzhugh, it would be circular to propose that. In an attempt to better understand the issues, pro and con, I briefly review the concepts of "explanation," "prediction," and "test." Karl Popper's writings predominate in this discussion, because Fitzhugh's claim of non-independence between cladogram and synapomorphy is couched in terms of Popperianism.

\section{DESCRIPTIVE GENERALIZATIONS}

Descriptive generalizations demand an explanation, and generalizations and explanations are of two basic

not exclude symplesiomorphy (salamander forelimbs and bird wings are homologous as tetrapod fore-appendages). Thus, in my view, synapomorphy and homology are not synonymous. 
kinds - what I will call universal and historical. Identifying the kind of descriptive generalization at hand depends on the general theory (e.g., law) that can be rationally justified as an explanation (Hull, 1974). Consider the following generalizations: (a) All atoms with an atomic number 79 are gold. (b) All swans are white. Atomic theory provides a universal type of explanation of the former descriptive generalization, whereas Darwinian theory provides an historical type of explanation of the latter. ${ }^{3}$ Why? Atomic theory is concerned with spatio-temporally unrestricted generalizations, the particular instances of which are historically independent, their identity being a matter of intentional definition (Frost and Kluge, 1994). Darwinian theory, on the other hand, pertains to spatio-temporally restricted things, such as organisms and species, which are connected by descent, and are therefore necessarily unique. Swans may then be understood as constituting a clade, which is defined extensionally in terms of feather color - the observed white feature having been "inherited" from a common ancestral white swan.

A universal explanation has long been thought of as a causal explanation, in so far as it is "an explanation of an explanation" (Mill, 1862: 510). Regarding historical explanation, as Mill put it,

"[a]n individual fact is said to be explained by pointing out its cause, that is, by stating the law or laws ... of which its production is an instance."

This is what has been called a singular event, contingent, or historical explanation. Thus, we have universal and historical descriptive generalizations and universal and historical explanations, correspondingly. Both kinds of explanations can be causal.

\section{A DEDUCTIVE MODEL OF HISTORICAL EXPLANATION}

In the main, Popper found Mill's distinction between universal and historical explanations quite acceptable; however, he sought to give greater precision to explanation, as indicated by the following quote (Popper, 1957: 122):

\footnotetext{
${ }^{3}$ Not withstanding the fact that "all swans are white" has been employed as a universal generalization (see discussion by Hull, 1974: 78).
}

\begin{abstract}
"I suggest that to give a causal explanation of a certain specific event means deducing a statement describing this event from two kinds of premises: from some universal laws, and from some singular or specific statements which we may call the specific initial conditions."
\end{abstract}

These premises are labeled $L$ and $C$, respectively, along with $E$ denoting the event, or effect, in a deductive model of explanation. Explanation is achieved by deducing effect from cause, in light of a law or some general theory. ${ }^{4}$ Summarizing,

$L$, explaining law $(\mathrm{s})$
$C$, specific initial condition(s) (cause)

$E$, specific event (effect).

\section{EVOLUTIONARY THEORY}

The general explanatory theory most commonly appealed to in phylogenetic systematics was framed by Darwin (1859). Darwin's theory can be summarized in terms of three inclusive evolutionary principles: (i) descent, (ii) with modification, and (iii) due to natural selection. ${ }^{5}$ It continues to surprise some that Popper accepted the scientific character of much, if not all, of Darwin's theory of evolution. Relevant to the current question, Popper (1957: 106-107; 1972; 1980) was explicit:

\footnotetext{
"What we call the evolutionary hypothesis is an explanation of a host of biological and paleontological observations-for instance, of certain similarities between various species and genera-by the assumption of common ancestry of related forms. ${ }^{2}$ This hypothesis is not a universal law, even though certain universal laws of nature, such as laws of heredity, segregation, and mutation, enter with it into the explanation. It has rather, the character of a particular (singular or specific) historical statement."
}

\footnotetext{
${ }^{4}$ The deductive-nomological model of Hempel and Oppenheim (1948) applies strictly to universal descriptive generalizations, where a genuine universal law is assumed necessarily. Moreover, those authors were especially concerned with the symmetry between explanation and prediction. My use of a similar deductive schema is special and self-contained, in that it is employed in a philosophical analysis of a particular kind of historical inference.

5"Phylogenetically evolved adaptations qua adaptations are the primary explanandum of natural selection, the central mechanism of neo-Darwinian theory" (Amundson, 1994: 560).
} 


\begin{abstract}
"2Feeling somewhat intimidated by the tendency of evolutionists to suspect anyone of obscurantism who does not share their emotional attitude toward evolution as a 'daring and revolutionary challenge to traditional thought,' I had better say here that I see in modern Darwinism the most successful explanation of the relevant facts."
\end{abstract}

I conclude from this quote, in addition to wholeheartedly endorsing neo-Darwinism, that Popper explained the historical descriptive generalization of certain shared similarities (synapomorphies) among terminal taxa in terms of inheritance (common ancestry), in light of the evolutionary principles of "descent, with modification."

Taking my cue from Popper (1957), I too will assume Darwin's principles of "descent, with modification," as the necessary explaining theories $(L)$ in a deductive historical model of explanation, with cladogram, and its common ancestral relations, constituting the specific initial condition $(C)$ and synapomorphy the specific event to be explained $(E)$. Summarizing:

$L$, descent, with modification

$C$, cladogram explanation

E, synapomorphy.

Defining the concept of homology as "features (parts, attributes) that were present in the common ancestor in which they are homologous" (Ghiselin, 1984), we are led to an inheritance model of explanation:

$L$, descent, with modification

$C$, cladogram

explanation of inheritance

$E$, synapomorphy as homology.

This definition of homology in terms of cladogram is not circular, because phylogeny is not defined in terms of homology (Ghiselin, 1966). Although this definition of homology may be sufficient ontologically, it contains no reference to operationalisms, such as the "similarity" of homologous adult features or their ontogenies.

Unknown to Popper is the fact that, in operationalizing the concept of homology, not all synapomorphies can be explained in terms of common ancestry. When two synapomorphies are incongruent, both cannot be interpreted as homologous - the shared-derived states of at least one of those two sets must have originated independently. From this fact one might derive a complementary model for homoplasy, which would imply that "descent, with modification," can also explain homoplasy:

\section{$L$, descent, with modification}

$C$, cladogram

explanation of independent origins

$E$, synapomorphy as homoplasy.

Complicating matters, Farris (1983) pointed out that while "descent, with modification," may be required in the inference of synapomorphy as homoplasy, those assumptions are not sufficient to explain synapomorphy as homology - an additional assumption(s) being required (Fig. 1). Further, in defining phylogenetic parsimony in terms of the "minimization of requirements for ad hoc hypotheses of homoplasy," Farris was simply following a well-known tenet of Popper's (e.g., 1983) philosophy of science-avoid all assumptions, except those required as background knowledge.

Farris' (1983) emphasis on homoplasy in the inference of phylogeny also followed Popper. Consider that the logical form of the conditional of cladogram/homology is modus ponens, if $\mathbf{p}$ then $\mathbf{q} / \mathbf{p} /$ therefore $\mathbf{q}$,
A

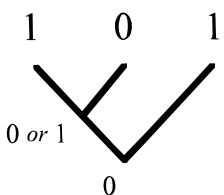

B

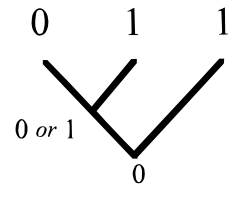

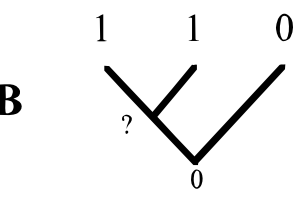

FIG. 1. "Descent, with modification," are sufficient assumptions in the inference of homoplasy, but not of homology (Farris, 1983). Let "descent" and "with modification" connote cladogram and synapomorphy, respectively. (A) Homoplasy in some form is deduced when conjoining a cladogram with an incongruent synapomorphy. (B) However, conjoining a cladogram with a congruent synapomorphy does not by itself lead to a deduction of homology, because the two assumptions do not rule out the possibility of parallelism. An additional assumption of evolutionary process is required to cover all cases of inference of two synapomorphous states being inherited from the same common ancestral condition, i.e., being homologous. 
whereas cladogram/homoplasy is the contrapositive conditional, modus tollens, if $\mathbf{p}$ then $\mathbf{q} /$ not- $\mathbf{q} /$ therefore not-p. Modus ponens is an invalid form of deductive inference, assuming just "descent, with modification," because the premises ( $L$ and $C$ ) do not preclude the "if not-p, then $\mathbf{q}$ " argument. To affirm the consequent (q) does not necessarily establish $\mathbf{p}$. The two possible deductive historical models for explaining synapomorphy may then be stated as:

Inheritance model of explanation (modus ponens: if $\mathbf{p}$ then $\mathbf{q} / \mathbf{p}$ /therefore $\mathbf{q}$ ):

$L$, descent, with modification

$L$, something more must be assumed

$C$, cladogram explanation of inheritance

$E$, synapomorphy as homology.

Independent origins model of "explanation" (modus tollens: if $\mathbf{p}$ then $\mathbf{q} /$ not- $\mathbf{q} /$ therefore not-p):

$L$, descent, with modification

$C$, cladogram

explanation of independent origins

$\boldsymbol{E}$, synapomorphy as homoplasy.

\section{POPPERIAN TESTABILITY}

Popperian testability provides a basis for understanding the concept of test as one of falsification. Testability involves the logical relationships between severity of test, $\mathbf{S}(\mathbf{e}, \mathbf{h}, \mathbf{b})$, and degree of corroboration, $\mathbf{C}(\mathbf{h}, \mathbf{e}, \mathbf{b})$,

$$
\mathbf{S}(\mathbf{e}, \mathbf{h}, \mathbf{b})=\mathbf{C}(\mathbf{h}, \mathbf{e}, \mathbf{b})=(p(\mathbf{e}, \mathbf{h b})-p(\mathbf{e}, \mathbf{b})) / \ldots,
$$

where $\mathbf{e}$ is evidence, $\mathbf{h}$ is hypothesis, and $\mathbf{b}$ is background knowledge. ${ }^{6}$ The significant numerator in Popper's expression of testability is the difference between the evidence with, $p(\mathbf{e}, \mathbf{h b})$, and without, $p(\mathbf{e}, \mathbf{b})$, the

${ }^{6} p$ in this context denotes propensity, a physical interpretation of the possibilities; $p$ does not pertain to precise probabilities, such as frequencies, which are abstractions (Popper, 1983: 286). The logical development of Popper's severity of test and degree of corroboration has been reviewed elsewhere (e.g., Kluge, 1997a). hypothesis, in light of the background knowledge. Put even more simply, testability is a measure of how much the hypothesis increases the likelihood of the evidence. Also noteworthy, regarding minimizing assumptions, the hypothesis receives a higher degree of corroboration the smaller the $p(\mathbf{e}, \mathbf{b})$ and in particular when $p(\mathbf{e}, \mathbf{b}) \ll 1 / 2$. If the evidence would seem to follow from background knowledge alone, then the hypothesis contributes little, and degree of corroboration is low.

\section{EXPLANATION AND EXPLANATORY POWER}

Farris (1983: 18) was the first to point out that " $[t]$ he explanatory power of a genealogy is ... measured by the degree to which it can avoid postulating homoplasies," and from which he derived the phylogenetic parsimony criterion, "the minimization of requirements for ad hoc hypotheses of homoplasy." When exercising this criterion, synapomorphies hypothesized as homologies are maximized on the cladogram that exhibits the fewest number of hypothesized evolutionary "modifications" (transformations, steps). According to Carpenter et al. (1998: 106), this connection between parsimony and explanation in testability was anticipated by Popper (1959: 401), who stated that

\section{"[f]or any given [evidence] $y, C(x, y)$ [the corroboration of theory}

$x$ by $y$ ] increases with the power of $x$ to explain $y$."

In explicating Popperian testability and Farris' argument for explanatory power in phylogenetic systematics, I have taken as standard practice the simultaneous analysis of a matrix of synapomorphies (see also Kluge, 1997a), where the matrix constitutes an array of evidence $\left(\mathbf{e}_{1}, \mathbf{e}_{2}, \mathbf{e}_{3}, \ldots \mathbf{e}_{\mathrm{n}}\right)$, and the number of terminal taxa on which the synapomorphies are scored determine the set of possible cladograms, an array of competing hypotheses $\left(\mathbf{h}_{1}, \mathbf{h}_{2}, \mathbf{h}_{3}, \ldots \mathbf{h}_{n}\right)$. Also, I assume "descent, with modification," as necessary background knowledge (b). Thus, for example, in the simple case of three terminal taxa, $\mathrm{A}, \mathrm{B}$, and $\mathrm{C}$, there are three possible rooted cladograms: $\mathbf{h}_{1},(\mathrm{~A}, \mathrm{~B}) \mathrm{C} ; \mathbf{h}_{2},(\mathrm{~A}, \mathrm{C}) \mathrm{B}$; and $\mathbf{h}_{3}$, $(B, C) A$. Further, given just the assumptions of "descent, with modification," (b), synapomorphies $\left(\mathbf{e}_{1}, \mathbf{e}_{2}, \mathbf{e}_{3}, \ldots\right.$ $\left.\mathbf{e}_{\mathrm{n}}\right)$ characteristic of the competing cladograms $\left(\mathbf{h}_{1}, \mathbf{h}_{2}\right.$, $\mathbf{h}_{3}$ ) should be equally likely, ceteris paribus. However, 
if a large majority of one of those possible classes of synapomorphies were to be discovered, for the sake of argument assume it is the class which characterizes $\mathbf{h}_{1}$, then this finding is unlikely given the background knowledge alone, but not under the background knowledge plus the postulated cladogram $((\mathrm{A}, \mathrm{B}) \mathrm{C})$. Therefore, $\mathbf{h}_{1}$ is the most highly corroborated, most severely tested of the three competing cladograms- $\mathbf{C}\left(\mathbf{h}_{1}, \mathbf{e}, \mathbf{b}\right)$ $>\mathbf{C}\left(\mathbf{h}_{2}, \mathbf{e}, \mathbf{b}\right)$ or $\mathbf{C}\left(\mathbf{h}_{3}, \mathbf{e}, \mathbf{b}\right)$, assuming only "descent, with modification." Further, in choosing the least falsified ${ }^{7}$ cladogram-according to Farris' phylogenetic parsimony criterion, minimizing requirements for ad hoc propositions of homoplasy $-\mathbf{h}_{1}$ is maximally explanatory, more synapomorphies being interpretable as homologues on $\mathbf{h}_{1}$ than on $\mathbf{h}_{2}$ or $\mathbf{h}_{3}$. This simple example also exposes the unnecessary nature of model assumptions in phylogenetic inference-the background knowledge of descent, with modification, being sufficient to make a rationally justified and meaningful explanation of homology of uniquely evolved historical generalizations.

\section{THE QUESTION OF INDEPENDENT TESTS}

Fitzhugh's (1998: 35) position can now be stated more precisely-if the causal condition of cladogram $(C)$ leads to an explanation of the current known effects of synapomorphy $(\boldsymbol{E})$, then synapomorphy (e) cannot be used as an independent test of competing cladograms $\left(\mathbf{h}_{1}, \mathbf{h}_{2}, \mathbf{h}_{3}, \ldots \mathbf{h}_{\mathbf{n}}\right)$. Although Fitzhugh stated the problem as if it were a fallacy of epistemological nonindependence, it is my contention that the reasoning is not logically erroneous (see also Hull, 1967). Rather, the apparent problem is simply a consequence of defining the possible effects of synapomorphy as a complement relation-that synapomorphy which is not due to a common ancestral origin (homology) must be of independent origin (homoplasy). An incongruent

\footnotetext{
${ }^{7}$ As with all singular descriptive generalizations, phylogenetic systematics can have only modest pretensions when it comes to falsification. For example, evidence in the form of synapomorphy can only constitute a weak form of falsification, because a synapomorphy test statement can only "count against" a cladogram, unlike a universal descriptive generalization being falsified by any one test (Hull, 1983; Sober, 1983: 339; contra Felsenstein, 1988: 529-530).
}

synapomorphy, homoplasy, constitutes falsifying evidence (e), and explanatory power is necessarily maximized in choosing the least falsified cladogram, because the congruent synapomorphies must be explained as having common ancestral origins (i.e., homologies). There seems to be no escape from this conclusion given just "descent, with modification," and employing the parsimony criterion of minimizing ad hoc hypotheses of homoplasy, which may be delimited according to the test of congruence.

However, if homoplasy is defined as independent origins, how can homoplasy be explained? Homoplasy cannot be explained in terms of "descent, with modification," because homoplasious states, by definition, constitute a spatio-temporally, historically unconnected, set of things. Although not to be confused with causal explanation, a possible explanation for homoplasy is simply one of "error," systematic and/or sampling. Further, as Popper (1972: 359) argued, if progress in science consists of trials "in the elimination of errors," then ways must be found to test synapomorphies as homoplasy, for otherwise phylogenetic systematics would not be progressive.

\section{PREDICTION}

If we can only adduce homoplasy as evidence counting against cladograms, and homology as coincidentally explained on the least disconfirmed hypotheses of sister-group relationships, we may feel that our explanation is circular and therefore quite unsatisfactory (Popper, 1972: 351). However, there remains the possibility of testing cladograms, and synapomorphies identified as homoplasies, according to prediction. Popper (1957: 124) clarifies such possibilities:

\footnotetext{
"... the use of a theory for predicting some specific event is just another aspect of its use for explaining such an event. And since we test a theory by comparing the events predicted with those actually observed, our analysis also shows how theories can be tested. Whether we use a theory for the purpose of explanation, of prediction, or of testing, depends upon our interest; it depends upon the question which statements we consider as given or unproblematic, and which statements we consider to stand in need of further criticism, and of testing."
}

Popper's (1957: 134) favorable reference to a systematic botany study (Frankel, 1941) illustrates how he believed observations guided by theory can lead to further tests. I have argued elsewhere (Kluge, 1997b) 
that independent testing in phylogenetic systematics lies in sophisticated falsification.

\section{SOPHISTICATED FALSIFICATION}

According to Lakatos (1993: 116), falsification must be "sophisticated," in the sense that there is an interplay between evidence (e) and competing hypotheses $\left(\mathbf{h}_{1}, \mathbf{h}_{2}, \mathbf{h}_{3}, \ldots \mathbf{h}_{\mathbf{n}}\right)$ that "leads to the discovery of novel facts." Such a progressive problem shift indicates a situation in which $\mathbf{h}_{1}$ suggests something more than $\mathbf{h}_{2}$ suggested, as well as suggesting more than is required by the data themselves.

As I have argued above, phylogenetic systematics may lay claim to explanation in testability, if only coincidentally in the "minimization of requirements for ad hoc hypotheses of homoplasy." However, that does not mean an increase in knowledge has been achieved, because all competing cladograms exhibit the same empirical content, $\mathbf{1}-p(\mathbf{h}, \mathbf{b})$. Therefore, for example, in the case of three competing cladograms, $\mathbf{C}\left(\mathbf{h}_{1}, \mathbf{e}, \mathbf{b}\right)$ may be judged the most severely tested and least falsified and having greater explanatory power than either $\mathbf{C}\left(\mathbf{h}_{2}, \mathbf{e}, \mathbf{b}\right)$ or $\mathbf{C}\left(\mathbf{h}_{3}, \mathbf{e}, \mathbf{b}\right)$. However, from this it does not follow that $\mathbf{C}\left(\mathbf{h}_{1}, \mathbf{e}, \mathbf{b}\right)$ also provides increased knowledge (sensu Lakatos, 1993).

Increased knowledge may, however, be claimed in phylogenetic systematics by making testable predictions from the most highly corroborated cladogram, where that particular hypothesis is an initial condition in its own logical deductive argument. Possible predictions and tests include the following (Kluge, 1997b). A re-analysis of certain characters predicted by the least falsified cladogram might be understood as explaining more in greater detail about synapomorphies. For example, the developmental and genetic distinctness of homoplasious states has the potential to be disconfirmed [e.g., in terms of the individualization studies of Wagner $(1989 a, b)]$. Further, we may even claim a test of evolutionary theory itself. For example, incongruences between the least falsified cladogram and best-fitting hierarchical hypotheses obtained from other historical sciences (e.g., the study of co-evolution, relative age of origin, and events of earth history) are disconfirmations of descent.

\section{DISCUSSION}

To be able to explain congruent synapomorphies as homologues on the most severely tested and least falsified cladogram is judged a consequence of defining homology and homoplasy as a complement relation, $a$ and not- $a$, respectively. However, in this sense, homoplasy is an abstraction and may be understood only nominally, e.g., as some minimal amount of error in the inference of homology. ${ }^{8}$ Of course, one could avoid having to propose error by providing a "real" definition of homoplasy, but that would require evoking a universal kind of explanation for a descriptive generalization of independently originating traits. I doubt many evolutionary biologists will entertain this possibility (however, see e.g., Lauder, 1982: 65; 1990: 332), because their focus is on historical explanations, as it is when they assume "descent, with modification." Moreover, the evolutionary biologist can always take the easy, unscientific way out and simply choose to explain homoplasious states as a set of homologues, i.e., as different synapomorphies characteristic of less inclusive groups of species. Of course, there is no test of error in this action.

In any case, only when phylogeny is known can it be said that the present effect of homology is necessarily deduced. Merely attempting to recognize the effects an hypothesis is intended to explain is not the same as actually having done so, as in the case of enumerative induction. Various operations in phylogenetic inference, such as defining homology and homoplasy as a complement relation, may lead to the appearance of epistemological dependence, and while those practices may be questioned (sensu Fitzhugh, 1998) they cannot be condemned as "viciously circular" (Hull, 1967: 177). I suggest the relationship between homology and homoplasy, at least potentially, is one of reciprocal clarification (Hennig, 1966); the more we test incongruent synapomorphies for error, the more our competing cladograms will be severely tested and the more our hypotheses of homology will be corroborated.

Science has been defined as a concern for explanation, prediction, and testing. I conclude that all three

${ }^{8}$ Coddington (1994) reached the same conclusion that some homoplasy is error. His argument was based on a statistical analogy, whereas my conclusion that all homoplasy is error follows from a deductive model of inference. 
criteria can be met when phylogenetic systematics is practiced according to Popperian testability (Popper, 1983) and the most-parsimonious cladogram is accepted as the working hypothesis from which testable predictions are made. Those predictions may include tests of homoplasy and descent. In these kinds of possibilities there is the independent testability of the premises, the statement of the explaining laws and specific initial conditions, which Popper (1972: 349) argued is the "very heart" of explanation.

\section{ACKNOWLEDGMENTS}

I thank Kirk Fitzhugh for criticizing my early attempts to understand the coherence and generality of phylogenetic systematics according to Popperian explanation, prediction, and test. Kirk's patience in explaining other philosophies of science to me has also been helpful. My understanding of Popperian testability has benefited considerably from discussions with Steve Farris and Mark Siddall. Jennifer Ast, Sharon Jansa, Maureen Kearney, and John Wenzel read the manuscript and offered valuable criticisms. I was also challenged on a number of points by Brian Crother and the members of his discussion group at Southeastern Louisiana University, Hammond. It goes without saying, however, that I am solely responsible for all the arguments and conclusions in this paper. The original version of the manuscript was completed at the Cladistics Institute, Harbor Springs, Michigan.

\section{REFERENCES}

Amundson, R. (1994). Two concepts of constraint: Adaptationism and the challenge from developmental biology. Phil. Sci. 61, 556-578.

Carpenter, J. M., Goloboff, P. A., and Farris, J. S. (1998). PTP is meaningless, T-PTP is contradictory: A reply to Trueman. Cladistics 14, 105-116.

Coddington, J. A. (1994). The roles of homology and convergence in studies of adaptation. In "Phylogenetics and Ecology" (P. Eggleton and R. I. Vane-Wright, Eds.), pp. 53-78. Linnean Symp. Ser., Academic Press, NY.

Darwin, C. (1859). "The Origin of Species by Means of Natural Selection, or the Preservation of Favoured Races in the Struggle for Life." John Murray, London. [(1964). Facsimile of the 1st ed. Harvard Univ. Press, Cambridge, MA]
Farris, J. S. (1983). The logical basis of phylogenetic analysis. In "Advances in Cladistics II" (N. I. Platnick and V. A. Funk, Eds.), pp. 7-36. Columbia Univ. Press, NY.

Felsenstein, J. (1988). Phylogenies from molecular sequences: Inference and reliability. Annu. Rev. Genet. 22, 521-565.

Fitzhugh, K. (1998). C(h,be): e $\neq$ synapomorphy. In "HENNIG XVII: 17th Meeting of the Willi Hennig Society," pp. 34-35. Univ. São Paulo, Brazil.

Frankel, O. (1941). Cytology and taxonomy of Hebe, Veronica and Pygmaea. Nature 147, 117-118.

Frost, D. R., and Kluge, A. G. (1994). A consideration of epistemology in systematic biology, with special reference to species. Cladistics 10, 259-294.

Ghiselin, M. T. (1966). An application of the theory of definition to systematic principles. Syst. Zool. 15, 127-130.

Ghiselin, M. T. (1984). "Definition," "character," and other equivocal terms. Syst. Zool. 33, 104-110.

Hennig, W. (1966). "Phylogenetic Systematics.” Univ. Illinois Press, Chicago.

Hempel, C., and Oppenheim, P. (1948). Studies in the logic of explanation. Phil. Sci. 15, 135-175.

Hull, D. L. (1967). Certainty and circularity in evolutionary taxonomy. Evolution 21, 174-189.

Hull, D. L. (1974). "Philosophy of Biological Science.” Prentice-Hall, Englewood Cliffs, NJ.

Hull, D. L. (1983). Karl Popper and Plato's Metaphor. In "Advances in Cladistics II” (N. I. Platnick and V. A. Funk, Eds.), pp. 177-189. Columbia Univ. Press, NY.

Kluge, A. G. (1997a). Testability and the refutation and corroboration of cladistic hypotheses. Cladistics 13, 81-96.

Kluge, A. G. (1997b). Sophisticated falsification and research cycles: Consequences for differential character weighting in phylogenetic systematics. Zool. Scripta 26, 349-360.

Lakatos, I. (1993). Falsification and the methodology of scientific research programmes. In "Criticism and the Growth of Knowledge" (I. Lakatos and A. Musgrave, Eds.), pp. 91-196. Cambridge Univ. Press, London.

Lauder, G. V. (1982). Historical biology and the problem of design. J. Theor. Biol. 97, 57-67.

Lauder, G. V. (1990). Functional morphology and systematics: Studying functional patterns in an historical context. Annu. Rev. Ecol. Syst. 21, 317-340.

Mill, J. S. (1862). "A System of Logic, Ratiocinative and Inductive," 5th ed. [in 2 vol.]. Parker, Son, and Bourn, West Strand, London.

Popper, K. (1957). “The Poverty of Historicism.” Routledge and Kegan Paul, London.

Popper, K. (1959). "The Logic of Scientific Discovery.” Harper and Row, NY. [1968 edition]

Popper, K. (1972). "Objective Knowledge: An Evolutionary Approach.” Clarendon Press, Oxford. 
Popper, K. (1978). Natural selection and the emergence of mind. Dialectica 32, 339-355.

Popper, K. (1980). Evolution. New Scientist 87, 611.

Popper, K. (1983). "Realism and the Aim of Science." Routledge, London. [1992 reprint]
Sober, E. (1983). Parsimony in systematics: Philosophical issues. Annu. Rev. Ecol. Syst. 14, 335-357.

Wagner, G. P. (1989a). The origin of morphological characters and the biological meaning of homology. Evolution 43, 1157-1171.

Wagner, G. P. (1989b). The biological homology concept. Annu. Rev. Ecol. Syst. 20, 51-69. 